\title{
Telemedicine For Eating Disorders: A Brief Report of A Italian Project
}

\author{
Giulia Savarese ${ }^{1}$, Luna Carpinelli ${ }^{1}$, Maria Rosaria Padovano ${ }^{1}$, Nadia Pecoraro ${ }^{1}$, Oreste \\ Fasano $^{1}$, Monica Mollo ${ }^{1}$, Claudio Di Giovanni ${ }^{1}$, Martino Politi ${ }^{1}$
}

1. Department of Medicine and Surgery, University of Salerno (Italy)

\begin{abstract}
The objectives of the present work are the research and development of a Telemedicine system for therapy and enhanced monitoring of Eating disorders (ED). To this end, useful tools have been developed both for patients and caregivers and for operators: a) a mobile application for medical and psychological updates on the status of patients and through which it is possible to access psychological counselling services for family caregivers; and b) virtual and immersive reality viewers for the therapy of ED patients with social anxiety.
\end{abstract}

Keywords: eating disorders, telemedicine, cyber-therapy, counselling, caregivers

\section{INTRODUCTION}

According to the European Health Telematics Observatory, which oversees the medical applications in telematics, telemedicine is 'the integration, monitoring and management of patients, as well as the education of patients and staff, using systems that allow prompt access to expert advice and patient information, regardless of where the patient or information resides' (De Moor et al., 1996). In Italy and abroad there are several telemedicine systems available for Eating disorders (ED). The team at Castelnuovo et al. (2012) have used telemedicine for the long-term treatment of obesity, noting that it contributed to or assisted body weight stabilization. Outside of Italy, the research group led by Schlegl et al. (2015) conducted a systematic study of the existing scientific literature to evaluate the potential of telemedicine to prevent and treat eating disorders (in particular for anorexia nervosa and bulimia nervosa) and to also assist the caregivers of patients with ED. The review was based on forty studies that provided evidence of the efficiency of e-therapy specifically for patients with bulimia nervosa. Encouraging results also emerged from Internet-based ED prevention and assessment programs for caregivers and patients, which involved a certain percentage of the population that is usually not reached, thus improving access to primary care. Moreover, Aardoom et al. (2016) have reported symptom reduction of ED in patients who have undergone self-help driven, Internet-based cognitive-behavioural therapy.

A study by Mitchell et al. (2008) has investigated the effectiveness and acceptability of cognitivebehavioural therapy (CBT) protocols for Bulimia nervosa (BN) with respect to a comparable therapy delivered via telemedicine. Participants in a randomized study performed $20 \mathrm{CBT}$ sessions for BN, over a span of 16 weeks, during which trained therapists offered homework exercises faceto-face (FTF-CBT) or through telemedicine (TV-CBT). The results were based on the frequency 
binge, and evaluated through a post-treatment interview and follow-up after 3 and 12 months. At the end of the treatment, only the levels of restriction and abstinence were higher in patients treated in FTF-CBT mode, although the differences were not statistically significant compared to those reported for the TV-CBT mode. By analysing the study of Mitchell et al. (2008), Ertelt et al. (2013), showed that there is a difference in the scores given by therapists and patients regarding treatment effectiveness in TV-CBT: patients tend to give significantly higher scores on the therapeutic outcome than the therapists because they perceive more positively the use of telemedicine in their treatment. Furthermore, Crow et al. (2009) have studied the effectiveness in terms of costs of telemedicine. The total cost per patient in FTF-CBT mode is $\$ 9324.68$, while in TV-CBT mode it is $\$ 7300.40$. The cost difference arises from the travel expenses of the therapist. Last, the review by Shingleton et al. (2013), which covers studies published from 2002 to 2012, shows that the use of diversified technologies to support CBT (e.g., teletext, e-mail, CD-ROM, Internet, text messages) improves the treatment of patients with ED (especially BN) at various levels of care (e.g., therapy, self-guidance, adjunctive therapy), although not without certain warnings for patients who have a more serious, pathological condition which requires greater interaction with the medical care providers.

Turning to the creation of specialized mobile applications (apps), there is a variety of existing studies (Bauer et al., 2013; Fairburn et al., 2015). In specific, one conducted by Faiburn et al. (2015) evaluated the clinical utility of 39 apps designed for patients with ED and 5 apps for professional specialists in the clinic sector, concluding that they could contribute to effective personalized treatments. Tregarthen et al. (2015) described the development and improvement of a free application for mobile phones which people with ED could use to self-monitor food intake, emotions, behaviour, and thoughts. The application also included positive reinforcement, tips on coping skills, social support, and feedback components. The application was downloaded by over 100,000 users over a period of two years. Among the users, almost 50\% said they had not been clinically diagnosed and 33\% reported not to have disclosed their eating disorder to anyone.

Telemedicine has also been used to support the family of the ED patient. Goldfield et al. (2003) have pointed out that most of the hospitals specialized in the treatment of eating disorders are only found in large urban centres, therefore not accessible to people living in rural communities underserved by transport as well as by social security systems. From the analysis of the clinical case of an adolescent with Nervous Anorexia (AN), recruited in a hospital treatment program in urban areas, the effectiveness of family therapy delivered through the use of Telemedicine was highlighted. The approach showed high levels of efficacy in the clinical recovery of the patient, measured based on objective (weight gain, improvement of medical conditions) and subjective (clinical observations) criteria. Furthermore, all family members reported great satisfaction with - without worrying about privacy. Anderson et al. (2017) analysed the cases of ten adolescents with AN involved in a study via a tele-health platform. The outcome of the treatment was determined using the median body mass percentage index (\% mBMI), the examination of eating disorder (EDE), the indices for depressive symptomatology, and the amount of self-esteem. The post-treatment mBMI percentage was significantly elevated at the 6-month follow-up ( $\mathrm{p}=0.032)$ compared to the baseline pretreatment score $(p=0.013)$. Similar results were achieved for the overall EDE score $(p=0.002$ in follow-up and 0.001 in baseline). 
Savarese, G., Carpinelli, L., Padovano, M. R., Pecoraro, N., Fasano, O., Mollo, M., Giovanni C. D., \& Politi, M. (2020). Telemedicine for Eating Disorders: A Brief Report of a Italian Project. Advances in Social Sciences Research Iournal, 7(4) 292-297.

\section{OBJECT}

The present project pertains to the research and development of a Telemedicine system for the therapy and monitoring of Eating disorders (ED). Our study proposes to identify the best methodology to deliver digital medical care, which can offer support to the care therapies of ED and to develop tools for patients, medical care providers, and caregivers that family members can also use. To this end, the following activities were implemented:

\section{App for the medical and psychological counselling for family caregivers}

In order to not only offer emotional and behavioural support to users who benefit from the therapy, but also allow the reporting of any reactions or abnormal behaviour of patients in real time. To this purpose, an ad hoc mobile application was created, which used video communication tools to carry out sessions, visits, consultations, and updates of clinical and instrumental data. Through the app you are able to remotely monitor any comorbidities (diabetes, arterial pressure, heart rate, and continuous ECG), weight, BMI, and the degree of patient compliance to nutritional, psychiatric, and psychological therapy.

\section{Design and implementation of virtual and immersive reality applications}

in order to start a restructuring of anxious and emotional disorders, in particular a ED patient with social anxiety.

\section{DESCRIPTION OF THE DESIGNED AND DEVELOPED TOOLS RELATED TO THE TARGET ACTIVITIES}

\section{App for the medical and psychological counselling for family caregivers and building an ad} hoc app

A mobile application has been created, whose structural characteristics satisfy the need for care and research. The app is also provided with an RSS feed, which can be fed with information and news on EDs via GOOGLE ALERT. Through the app it is possible:

- to choose, for the member of the care community (briefly hereinafter MCC), the medical field in which the requested support is placed;

- find the appropriate Counsellor, through the function 'study secretary' (hereinafter SDS), communicating his/her availability to the MCC, by indicating of the day and time when it would be possible to provide the service; once the availability of the applicant is received, the SDS confirms the appointment to the Counsellor;

- provide the Counsellor, through the functions of the platform, full access to the patient's medical record;

- provide the MCC with access to therapeutic indications to facilitate possible requests for clarifications or indications on the methods of taking the medication/receiving therapy.

The interview between the Counsellor and MCC takes place through the most suitable channel, including:

- Instant messaging (WhatsApp)

- Video calling (WhatsApp)

- Voice calling (normal phone call) 
The SDS has a series of functions available for consulting any received requests for counselling and for assignment to counsellors. The diary is a simple function which can be used to record any notes on the content of the counselling session, which may be used by different successive Counsellors.

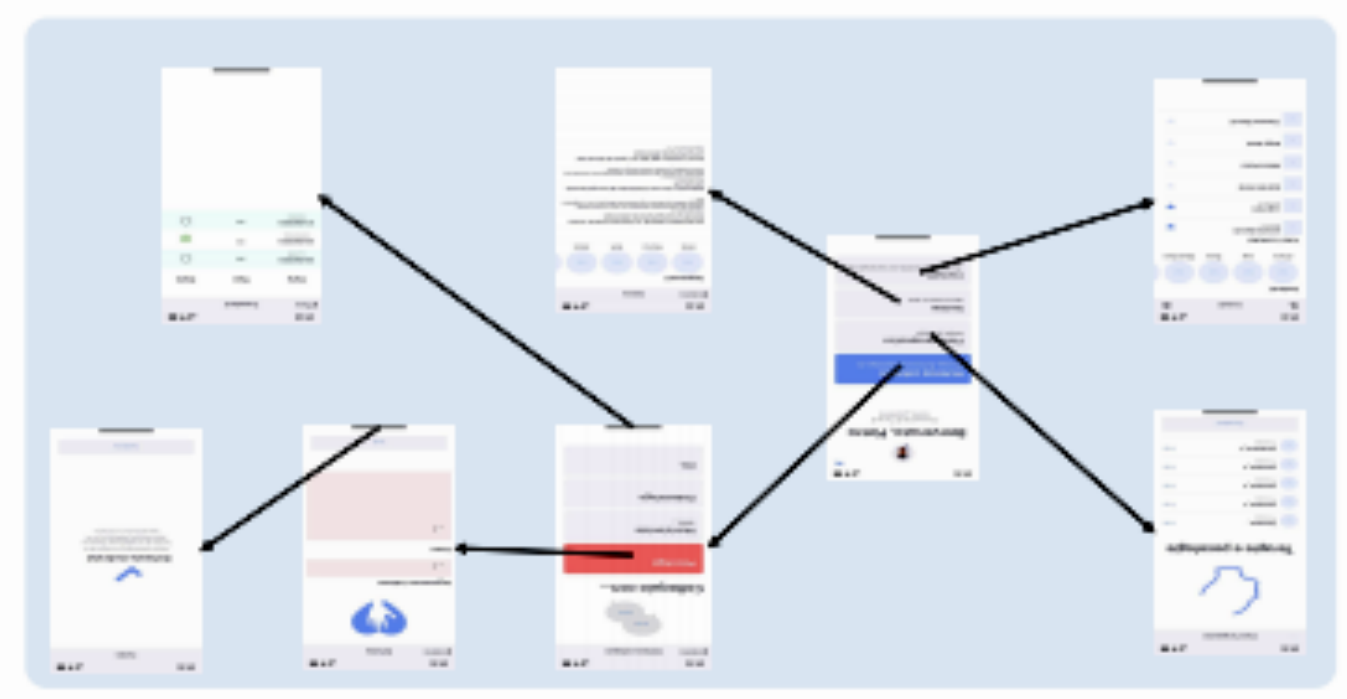

Diagram : Entity relationship diagram

The MCC has a very simple interface, with a choice of options on the Home screen (see DIAGRAM):

- 'DO YOU NEED HELP?'

- 'DO YOU WANT TO SEE THE THERAPIES IN ACT?'

- 'DO YOU WANT TO READ THE LATEST ED INFORMATION?'

- 'DO YOU NOT REMEMBER THE CONTACTS?'

TAB 1: clicking on 'DO YOU NEED HELP?' opens a page that contains the TABs, e.g., wicked: NUTRITION, THERAPIES IN ACT, BEHAVIOR, etc. An extensive education phase was part of the project, in presence of medical care providers who clearly explained to the patients what each area entails, in order to avoid wasting time and misunderstanding. From this list you can send requests for different online services.

TAB 2: the pharmacological or behavioural therapies in use with the possible dosages and recommendations are displayed.

TAB 3: a simple feed interface is provided.

For 'DO YOU NOT REMEMBER THE CONTACTS?', the name of the referred therapist is provided (which can also be communicated to the Counsellor) as well as the telephone number of the SDS. The SDS function has the ability to send a reminder SMS to the MCC.

An example interaction scenario is presented below:

1. The MCC accesses the 'DO YOU NEED HELP?' section of the app;

2. The MCC selects one of the sections (e.g., NUTRITION); 
Savarese, G., Carpinelli, L., Padovano, M. R., Pecoraro, N., Fasano, O., Mollo, M., Giovanni C. D., \& Politi, M. (2020). Telemedicine for Eating Disorders: A Brief Report of a Italian Project. Advances in Social Sciences Research Iournal, 7(4) 292-297.

3. The app provides a form for entering a service request;

4. The MCC compiles the request briefly indicating the reason for the request;

5. The MCC sends the request;

6. The SDS accesses the platform and consults the list of requests, which includes the request made in point 5;

7. The SDS consults the request made in point 5 and verifies the availability of the interested counsellor;

8. The SDS contacts the MCC (via message, notification, or call) to confirm the appointment that will take place according to the agreed times and procedures;

9. 8a. The SDS confirms the appointment on the platform;

10. The MCC receives an appointment confirmation message. The confirmation is also available on the app.

\section{Design and implementation of virtual and immersive reality applications}

The instrument used was Oculus Go, a very versatile and technologically advanced visualization tool equipped with earphones, while two video products were used for video shooting: Gopro0mni 360, which is supported by 6 synchronous cameras and Panasonic AW-360C10, a high-profile professional machine, also equipped with a microphone for audio recording. The implemented system allows you to manage videos not only through the viewer, but also on a smartphone or iPad through a specific app or directly on a PC through a browser. Imagined based on previous meetings with a team of psychotherapists, 3 scenarios with 4 corresponding videos were conceived (there are two videos for the first scenario). The duration of each video is around 3 minutes, and 'relaxing' introductions could also be integrated in the future.

\section{Scenario 1: Aula Quasar}

VIDEO 1a: corridor outside a classroom showing people (typically students) entering the classroom and taking their seats

VIDEO 1b: shows the inside of the half-empty classroom that gradually fills up with people, focusing on a student who is called by the professor to take an exam. The classroom gradually begins to empty as students leave.

\section{Scenario 2:}

The Place ("Campo dei Fiori"), which can be compared to a scenario with various people moving around, either few or many

VIDEO 2: a time-lapse of the square in the afternoon, which consists of a compressed video of a shot that took place over a long period of time. In this way, the video, even at accelerated speed, allows a wide number of movements and events to be filmed within the allocated space. You can opt for the time-lapse and/or a short extract of frames in normal time.

\section{Scenario 3: District "Mandrione"}

VIDEO 3: shot from a point near the bar area. Empty at first, it gradually fills up with people who order, drink, or chat. 
A relaxing introductory part was also created, consisting of soft music accompanied by still images in the background, which can help the patient prepare for the medical visit.

\section{CONCLUSIONS}

Based on the existing studies in the scientific literature on the positive value of telemedicine applied to caring for and treating ED patients and with the aim of using telemedicine as an accompaniment to the treatment and care of patients and as an aid for family/caregivers, a series of tools have been developed: a) an app for medical and psychological counselling of patient caregivers, with the participation of different care professionals; b) virtual and immersive reality viewers for short cyber-therapy sessions for ED patients showing prevalent clinical features of social anxiety. The trial is in progress and the evaluated results are still preliminary.

\section{References}

Aardoom, J. J., Dingemans, A. E., \& Van Furth, E. F. (2016). E-health interventions for eating disorders: emergingfindings, issues, and opportunities. Current psychiatry reports, 18(4), 42.

Anderson, K. E., Byrne, C. E., Crosby, R. D., \& Le Grange, D. (2017). Utilizing Telehealth to deliver family-based treatment for adolescent anorexia nervosa. International Journal of Eating Disorders, 50(10), 1235-1238.

Bauer, S., \&Moessner, M. (2013). Harnessing the power of technology for the treatment and prevention of eating disorders. International Journal of Eating Disorders, 46(5), 508-515.

Castelnuovo, G., Corti, S., Cuzziol, P., Scalia, M. T., \& Manzoni, G. M. (2012). Il ruolo della telemedicina per il trattamento a lungo termine dell'obesità: Il progetto TECNOB. In Clinica psicologica dell'obesità (pp. 219-236). Springer, Milano.

Crow, S. J., Mitchell, J. E., Crosby, R. D., Swanson, S. A., Wonderlich, S., \&Lancanster, K. (2009). The cost effectiveness of cognitive behavioral therapy for bulimia nervosa delivered via telemedicine versus face-to-face. Behaviourresearch and therapy, 47(6), 451-453.

De Moor, G., Laires, M., McCurry, M., \& Thayer, C. (1996). The European health telematics observatory. STUDIES IN HEALTH TECHNOLOGY AND INFORMATICS, 108-112.

Ertelt, T. W., Crosby, R. D., Marino, J. M., Mitchell, J. E., Lancaster, K., \& Crow, S. J. (2011). Therapeutic factors affecting the cognitive behavioral treatment of bulimia nervosa via telemedicine versus face-to-face delivery. International Journal of Eating Disorders, 44(8), 687-691.

Fairburn, C. G., \&Rothwell, E. R. (2015). Apps and eating disorders: A systematic clinical appraisal. International Journal of Eating Disorders, 48(7), 1038-1046.

Goldfield, G. S., \&Boachie, A. (2003). Case report: delivery of family therapy in the treatment of anorexia nervosa using telehealth. Telemedicine Journal and e-health, 9(1), 111-114.

Mitchell, J. E., Crosby, R. D., Wonderlich, S. A., Crow, S., Lancaster, K., Simonich, H., ... \& Myers, T. C. (2008). A randomized trial comparing the efficacy of cognitive-behavioral therapy for bulimia nervosa delivered via telemedicine versus face-to-face. Behaviou research and therapy, 46(5), 581-592.

Schlegl, S., Bürger, C., Schmidt, L., Herbst, N., \&Voderholzer, U. (2015). The potential of technology-basedpsychological interventions for anorexia and bulimia nervosa: a systematic review and recommendations for future research. Journal of medical Internet research, 17(3), e85.

Shingleton, R. M., Richards, L. K., \& Thompson-Brenner, H. (2013). Using technology within the treatment of eating disorders: A clinicalpractice review. Psychotherapy, 50(4), 576.

Tregarthen, J. P., Lock, J., \&Darcy, A. M. (2015). Development of a smartphone application for eating disorder selfmonitoring. International Journal of Eating Disorders, 48(7), 972-982. 